ACTA UNIVERSITATIS LODZIENSIS

Folia Litteraria Romanica 15, 2020

https://doi.org/10.18778/1505-9065.15.19

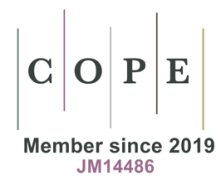

\author{
Marta Elżbieta Trębska \\ Université de Lodz / Université de Nantes \\ marta.trebska@edu.uni.lodz.p1
}

\title{
Du titre à la poétique : les recueils de nouvelles de Benoît Gonon*
}

\begin{abstract}
RÉSUMÉ
Benoît Gonon, auteur de trois recueils de récits brefs, est aujourd'hui presque complètement inconnu. Étant l'un des épigones de la nouvelle exemplaire au XVII ${ }^{\mathrm{e}}$ siècle, il écrit ses histoires pour moraliser et édifier ses destinataires. Il n'est guère surprenant que, au niveau des thèmes abordés par l'auteur, son œuvre ne révèle pas trop d'originalité. Cependant, on observe chez lui un élément intéressant : les titres qu'il donne à ses recueils de nouvelles et qui contiennent en germe son programme littéraire. Dans chaque volume il se présente comme un compilateur qui cherche l'invention chez d'autres écrivains. Mais, d'un volume à l'autre, Benoît Gonon ajoute aussi d'autres éléments intéressants : il enrichit progressivement la couche thématique de ses recueils, il signale son public visé. Ces amplifications du titre révèlent la préoccupation de notre célestin pour l'efficacité rhétorique des titres de ses recueils.
\end{abstract}

MOTS-CLÉS : nouvelle exemplaire, histoire, Benoît Gonon, titres, XVII ${ }^{\mathrm{e}}$ siècle, littérature française

\section{"From Title to Poetics: Benoît Gonon's Collections of Short Stories"}

\section{SUMMARY}

Benoît Gonon, author of three collections of short stories, is almost completely unknown today. As one of the epigones of the genre of short story in the 17th century, Gonon writes his stories to moralize and edify his readers. It is hardly surprising that, in terms of the themes addressed by the author, his work does not reveal too much originality. However, one observes there an interesting element: the titles given by Gonon to the three volumes of short stories, which contain in germ the literary program of the author. In each volume Gonon introduces himself as a compiler seeking invention from other writers. But, from one volume to another, Benoît Gonon also adds other interesting elements:

* Cet article s'inspire de notre mémoire de maîtrise « Les nouvelles exemplaires du révérend père Benoît Gonon : étude historique littéraire et édition de fragments choisis », rédigé sous la direction du professeur Witold Konstanty Pietrzak, Université de Łódź, 2016. Dans ce mémoire nous avons consacré quelque place à l'analyse des titres. Ici, nous envisageons d'approfondir cette problématique. 
he gradually enriches the thematic layer of his compilations, he signals his target audience. These amplifications of the title reveal the concern of the author for the rhetorical effectiveness of the titles of his compilations.

KEYWORDS - short exemplary story, history, Benoît Gonon, titles, $17^{\text {th }}$ century, French literature

Actif dans la première moitié du XVII siècle, Benoît Gonon, auteur de trois volumes de nouvelles ${ }^{1}$, est aujourd'hui presque entièrement inconnu. L'une des raisons de cet oubli est l'opinion des chercheurs, notamment de Sergio Poli et René Godenne, qui, en comparant l'art de Gonon, par exemple, avec celui de Jean-Pierre Camus ou celui de François de Rosset, ont trouvé peu d'intérêt dans les thèmes abordés par l'écrivain ${ }^{2}$. Cependant, il existe un aspect de cette œuvre qui révèle une originalité indéniable, à savoir les titres des recueils. Au XVII ${ }^{\mathrm{e}}$ siècle - et l'on pourrait démontrer le même phénomène pour le cinquecento - rares sont les auteurs qui publient plusieurs recueils de nouvelles et qui en différencient les titres. François de Rosset fait paraître en 1614 ses Histoires mémorables et tragiques de nostre temps dont les rééditions, certes augmentées, porteront le même titre. Les Tragiques accidents des hommes illustres de Pierre Boitel paru en 1616 est un ouvrage qui connaîtra en 1622 une deuxième édition, également augmentée, avec un titre nouveau, Le Théâtre tragique. À l'opposé de ces écrivains qui ne font qu'enrichir le corpus initial de leurs récits, nous découvrons la production de Jean-Pierre Camus. L'évêque de Belley multiplie ses recueils d'histoires dévotes en leur donnant toujours un titre nouveau et sans reprendre d'un recueil à l'autre les mêmes histoires comme le font Rosset et Boitel. Occurrences remarquables, Spectacles d'horreur ou Rencontres funestes - voici juste quelques exemples de titres qui révèlent un véritable génie. Mais, à l'ombre de Camus, on rencontre aussi Simon Goulart, qui,

1 Histoires veritables et curieuses, où sont representées les étranges avantures des personnes Illustres : Recolligées de plusieurs celebres Historiens : Par le R. Pere Benoist Gonon, Celestin de Lyon, Lyon, Jaques du Creux, 1644 ; Histoires pitoyables et tragiques, où les actions vertueuses et vicieuses de quelques illustres Payens et Chrestiens sont amplement representées. Recueillies de plusieurs celebres Historiens. Par le R. P. Benoist Gonon, Celestin de Lyon, Lyon, Claude de la Rivière, 1646 ; Histoires admirables, où les étranges avantures, vertus, vices et misères de plusieurs Empereurs, Roys, Princes, Grands Seigneurs, et autres Personnes considerables sont amplement representées. Pour l'instruction et divertissement de toutes sortes de personnes. Recueillies de plusieurs celebres Autheurs, par le Reverend Pere Benoist Gonon, Celestin de Lyon, Lyon, Claude de la Rivière, 1653.

2 S. Poli, Storia di storie. Considerazioni sull'evoluzione della storia tragica in Francia dalla fine delle guerre civili alla morte di Luigi XIII, Genova, Publicazioni dell'Istituto di Lingue e letterature straniere moderne, 1985, p. 326-327 ; R. Godenne, Histoire de la nouvelle française aux XVII et XVIII siècles, Genève, Droz, 1970, p. 40. G. Hainsworth, Les « Novelas exemplares » de Cervantes en France au XVII' siècle », Paris, Champion, 1933, p. 106-108, et N. Cremona " Pleines de chair et de sang " : poétique d'un " genre à succès », l'histoire tragique, thèse de doctorat, Paris, 2009, p. 441, voient dans le style assez simple et privé d'ornements rhétoriques de Gonon l'une des caractéristiques qui rapprochent son œuvre narrative d'une chronique. 
entre 1600 et 1610, publie plusieurs volumes d'Histoires admirables et memorables de nostre temps. Le titre de ces volumes ne change pas, alors que la matière, comme chez Camus, en est toujours nouvelle. C'est dans le contexte de ces deux écrivains qu'il faut replacer l'œuvre narrative de Benoît Gonon. Tous les trois ont le mérite de l'invention, mais chacun a sa propre manière de formuler le titre. Si Camus semble indépassable sur ce point, la comparaison avec Goulart paraît a priori bien plus profitable. En effet, les titres de recueils de Benoît Gonon se composent d'un substantif invariant - « histoires »-accompagné de différents qualificatifs et suivi d'éléments variables, et ainsi contiennent en germe le programme littéraire du nouvelliste. Dans cet article, nous allons analyser ces titres sous l'angle de leur morphologie, de leur fonctionnalité et de leur sémantique pour cerner le caractère générique des récits gononiens.

\section{Esquisse bio-bibliographique}

D'après les notices laconiques des historiens ${ }^{3}$, Benoît Gonon est né à Bourg-enBresse, probablement vers 1580 . La date qui ne prête pas à controverse est celle de sa consécration dans le couvent : le 4 avril 1608. On sait aussi qu'il est décédé à Lyon en 1656. Selon ces historiens, il était un « cellulae amantissimus » et un « homme de prière et d'oraisons », et ceci explique sans doute la paucité des détails sur sa vie. Néanmoins nous connaissons la liste des œuvres de Benoît Gonon qui permet de se faire une certaine idée de ses intérêts d'homme de lettres. On peut ainsi observer deux périodes dans son activité littéraire : latine et française. Sa première publication remonte à 1625 , ce qui montre qu'il a trouvé sa vocation d'écrivain assez tard, à l'âge de quelque 45 ans; elle porte le titre Uitae \& sententiae Patrum Occidentis, libris septem digestae, necnon ex antiquis msc. Codd \& Ecclesiarum Breuiariis collectae, \& annotationibus selectis exornatae. Nous ignorons la réception de cet in-folio d'hagiographie de plus que 600 pages en France, mais nous savons qu'il a suscité un certain intérêt en Europe, comme en témoigne la traduction polonaise ${ }^{4}$. Ensuite, il a sans doute travaillé simultanément sur deux autres œuvres publiées en 1635 : Historia Eucharistica, duobus libris descripta, in quibus multa egregia continentur, quae ad hoc praestantissimum Sacramentum amandum, adorandum, dignéque fumendum excitare possunt, qui aborde l'histoire de la doctrine catholique, et Schola sanctorum Patrum, in qua ex eorum dictis ac sententis modus acquirendi

3 A. Becquet, Gallicae Celestinorum congregationis ordinis S. Benedicti monasterium fundationes uirorumque uita aut scriptis illustrum, elogia historica, Paris, Florentinum Delaulne, 1749, p. 203-205 ; M. Depéry, Biographie des hommes célèbres du département de l'Ain, Bourg, P.-F. Boittier, 1833, p. 290.

4 Żywoty y nauki świętych pustelników zachodnich przez wielebnego oyca Benedykta Gonona Burgensa oyców Celestinów zakonnika wydane y zebrane a językiem łacińskiem napisane; manuscrit 2958/1, Dolnośląska Biblioteka Cyfrowa. 
perfectionem edocetur, une compilation de sentences et de biographies des saints. Il est vraisemblable qu'à cette époque il se consacre aussi à la rédaction de ses œuvres inédites ${ }^{5}$. Ce travail en silence peut expliquer pourquoi une décennie passe entre la publication des Uitae \& sententiae Patrum Occidentis et l'Historia Eucharistica. En 1637, il fait sortir deux ouvrages sur la Vierge : Chronicon Deiparae Virginis Mariae et Viridarium Deiparae Virginis Mariae. À partir de cette époque Gonon n'écrit plus qu'en français. D'abord, en 1639, il donne une traduction de son premier ouvrage sur la mère de Dieu, Histoire et miracles de Notre-Dame, puis deux autres ouvrages de piété ${ }^{6}$ Enfin, entre 1644 et 1653 il fait paraître ses trois recueils de récits brefs, objet de notre article.

\section{2. Éléments de titrologie}

Avant d'entamer notre étude proprement dite, il est utile de rappeler la problématique de la titrologie en général. Depuis l'article de Claude Duchet $^{7}$ les critiques qui étudient les titres se sont multipliés, mais pour la plupart ils se concentrent sur les productions modernes. Chez Leo H. Hoek et Gérard Genette nous découvrons une approche théorique du titre qui, en raison de son caractère universel, semble plus intéressante de notre point de vue. Le premier ${ }^{8}$ envisage le titre comme un signe appartenant à un système de pratiques de communication sociale basées sur le texte. Déterminé par sa syntaxe, le titre y entre dans un vaste réseaux de relations qui définissent ses fonctionnalités diversifiées. Perçu de cette manière, il devient l'objet d'un modèle général, délibérément abstrait de tout contexte historique. Gérard Genette ${ }^{9}$, à son tour, se propose un but plus modeste, car, s'appuyant sur de nombreux exemples pris à la littérature non seulement moderne mais aussi plus ancienne, il entend décrire plusieurs critères qui permettent de saisir la spécificité du titre. Cette approche qui nous paraît plus pertinente pour l'étude des titres de Benoît Gonon, c'est pourquoi nous allons maintenant en rappeler les grandes lignes.

Le critique distingue plusieurs critères selon lesquels il envisage cette partie du paratexte : la structure, le lieu, le moment, le destinateur et le destinataire, et enfin les fonctions. Quant à la structure, le titre peut comporter trois éléments : le titre propre,

5 À savoir : Breuis historia Caelestinorum Galliae, histoire du couvent des célestins en France ; Tabella Passionis Christi, traité spirituel sur la vie et la mort de Jésus-Christ ; et enfin Chorus Sanctorum \& Beatorum diuersarum nationam, recueil de biographies des saints de diverses nations.

6 Les Illustres Penitens, et charitables envers les pauvres, avec l'histoire de Cariton, Lyon, Pierre Anard, 1641 ; La Chasteté récompensée, ou l'histoire de sept pucelles doctes, et sçavantes, Bourg en Bresse, Jean Tainturier, 1643.

7 Cl. Duchet, "La Fille abandonnée et La Bête humaine, éléments de titrologie romanesque », Littérature, Année 1973, n 12, p. 49-73.

8 L. H. Hoek, La Marque du titre : dispositifs sémiotiques d'une pratique textuelle, La Haye, Mouton, coll. «Approaches to semiotics », 1981.

9 G. Genette, Seuils, Paris, Éditions du Seuil, 1987, p. 54-97. 
le sous-titre et l'indication générique. Le critère du lieu touche à l'emplacement du titre dans le livre : sur « la première de couverture, le dos de couverture, la page de titre, et la page de faux titre $»^{10}$. Le moment où apparait le titre correspond à la date de publication de l'édition princeps. Le destinateur de droit du titre n'est pas forcément le même que celui de fait, ce dernier pouvant venir de l'éditeur ou de la critique postérieure de l'œuvre. Une autre différenciation s'impose quand on envisage le destinataire du titre qu'il ne faut pas confondre avec le destinataire de l'œuvre, le premier, par exemple le libraire ou le bibliothécaire, pouvant très bien se passer de la lecture du livre, superflue dans l'exercice de son métier. Le dernier critère, les fonctions, n'est pas le moins intéressant. Chose évidente, le titre constitue un label littéraire assurant à l'œuvre le statut d'un produit unique parmi tous les autres, et cette fonction identificatrice est, selon Gérard Genette, la seule indispensable. Ensuite, la fonction descriptive permet au titre de donner une certaine idée du livre ; en particulier, la caractéristique thématique en suggère la matière, tandis que la caractéristique rhématique permet moins d'attirer l'attention sur la forme que de saisir « le texte lui-même considéré comme œuvre et comme objet ${ }^{11}$. Mais, outre la capacité à produire un sens univoque, le titre possède aussi celle à connoter, à dire de manière implicite quelque chose de plus que ce que les paroles désignent directement. Sur la fonction de séduction, «à la fois trop évidente et trop insaisissable $\rangle^{12}$, le critique se prononce avec plus de sobriété, en pensant qu'il est impossible de mesurer la part du charme exercé par le titre dans le succès éditorial du livre que ce dernier est censé faire vendre.

\section{Analyse des titres de recueils de Benoît Gonon}

En partant de ces données, penchons-nous sur les titres gononiens. Puisque, d'une part, certains critères de Gérard Genette n'ont évidemment pas d'intérêt scientifique pour leur étude (par exemple, le lieu ou la fonction identificatrice) et que, d'autre part, le nouvelliste passant d'un volume de ses récits à l'autre enrichissait leurs titres, nous nous proposons d'organiser notre recherche autour de leur structure pour en déterminer les éléments variants et invariants.

\subsection{Indications génériques}

On remarque dans un premier temps que les titres gononiens contiennent tous un élément rhématique invariant qui est en même temps une indication générique : ils commencent par le mot « histoires ». Avec le choix de ce genre narratif l'auteur

\footnotetext{
${ }^{10}$ Ibid., p. 63.

${ }^{11}$ Ibid., p. 75.

${ }^{12}$ Ibid., p. 87.
} 
s'inscrit dans la tradition du récit bref. Parmi les différentes formes de ce dernier l'histoire doit sa spécificité à son rapport à la réalité, exigeant que le discours soit vrai, c'est-à-dire conforme à la substance des événements extralittéraires. En particulier, Gonon renoue avec la convention du récit établie à partir de l'Heptaméron de Marguerite de Navarre et poursuivie entre autres par Pierre Boaistuau et François de Belleforest ${ }^{13}$, et plus tard par Simon Goulart ou François de Rosset. Chez tous ces écrivains, le topos de la véracité, compromis par bien des nouvellistes depuis le Moyen $\hat{A g g e}^{14}$, retrouve sa gravité et sa justification morale.

Or, dans les titres gononiens, le terme invariant d'histoire s'accompagne toujours d'un autre élément rhématique, c'est-à-dire de l'adjectif qualificatif. Ce dernier exprime différentes propriétés des récits que nous allons envisager maintenant.

Ainsi, le premier volume de notre célestin est consacré au récit « véritable » et « curieux ». Le qualificatif " véritable », associé au terme générique d'histoire, véritable elle-même, crée certes un effet de redondance, largement accepté dans les milieux humanistes. Il est bien moins intéressant que le deuxième adjectif, " curieux ». Ce dernier désigne à la fois la singularité de la matière narrative et l'intérêt pour le lecteur. Les syntagme « histoire curieuse » n'est enregistré ni dans le Thresor de la langue françoyse de Nicot, ni dans le Dictionnaire universel de Furetière. Dans les titres de l'époque il n'apparaît qu'occasionnellement, par exemple l'Histoire curieuse de tout ce qui s'est passé à l'entrée de la Reyne (1635) par Jean Puget de la Serre ou Le Voyageur inconnu, histoire curieuse, \& apologetique pour les religieux (1630) par Jean-Pierre Camus. Enfin, l'utilisation de ce terme dans les titres de recueils est rare, car, comme l'observe Sergio Poli, les nouvellistes ont tendance à utiliser des épithètes consacrées telles que « admirable », « mémorable » ou « remarquable », qui portent souvent le même sens : «étrange $»^{15}$.

Dans le même temps, la notion de curiosité pose quelques problèmes à cause de sa mauvaise réputation, originaire du récit biblique où Ève, tentée par le serpent, brise le commandement divin et, malgré l'interdiction, mange le fruit de l'arbre de la connaissance du bien et du mal. La curiosité n'y est pas forcément un péché, mais plutôt une force générant la transgression de la loi du Seigneur. La volonté de connaître le monde n'est pas pourtant un acte nécessairement mauvais : c'est pour cela que Thomas d'Aquin introduit la distinction entre la curiosité et la studiosité qui désigne la capacité et la disposition humaine à rechercher le savoir en entrant en contact avec le Logos de Dieu ${ }^{16}$.

${ }^{13}$ W. K. Pietrzak, Le Tragique dans les nouvelles exemplaires en France au XVI siècle, Łódź, Wydawnictwo Uniwersytetu Łódzkiego, 2006, p. 62-69.

${ }^{14}$ K. Kasprzyk, Nicolas de Troyes et le genre narratif en France au XVI siècle, Warszawa / Paris, Państwowe Wydawnictwo Naukowe / Librairie C. Klincksieck, 1963, p. 326-329.

${ }^{15}$ S. Poli, op. cit., p. 31.

${ }_{16}$ Thomas d'Aquin, Somme théologique, IIa IIae, trad. E. Belin, Paris, Librairie Ecclésiastique et Classique, 1856, question 167 « De la curiosité », p. 213-225. 
Si en général la curiosité dénote l'appétit de savoir, il faut néanmoins remarquer que ce mot possède un sens double : objectif, où il désigne la qualité inhérente d'une chose qui se distingue des autres par son caractère singulier et qui mérite donc d'être mémorisée par l'écriture ou au musée, héritier des anciens cabinets de curiosités ; et subjectif, où la curiosité signifie aussi l'effet produit par cette chose sur celui qui la perçoit, c'est-à-dire la disposition de l'esprit qui se penche sur elle avec intérêt. Les histoires curieuses sont donc susceptibles d'éveiller l'esprit du lecteur en lui représentant la diversité du monde et des destins humains, de lui conférer une certaine vivacité, de le tenir en alerte sans pourtant lui inspirer des émotions fortes, véhémentes : celles-ci, comme nous allons le voir, sont le privilège des histoires admirables.

L'adjectif «tragique », utilisé par Gonon pour caractériser les récits de son deuxième volume, renvoie bien sûr à l'histoire tragique, genre inauguré par Pierre Boaistuau en 1559. Au XVII ${ }^{\mathrm{e}}$ siècle, ce genre est toujours pratiqué par les écrivains, et Sergio Poli dans sa monographie déjà citée en étudie les hauts et les bas. Il faut cependant préciser que, pour les littérateurs actifs en temps des guerres civiles en France, une histoire tragique devait être une chose bien différente de ce qu'elle deviendra pour les auteurs une centaine d'années plus tard. Transposition poḯtique de l'expérience vécue au quotidien pour les uns, elle ne sera plus qu'un souvenir lointain des malheurs passés, tout au plus un avertissement aux lecteurs contemporains, une pure res litteraria, pour les autres. Quand il donne à ses lecteurs un recueil d'histoires tragiques, c'est moins pour leur faire sentir leur condition d'individus ancrés dans le sillage du sanglant passé de leur pays que pour leur faire connaître le probable destin de l'homme, le fatum des Grecs, le leur propre. « Ces Histoires [...], écrit-il, feront cognoistre qu'il n'y a rien sous le Ciel, qui ne soit sujet à divers changemens : ce qui appert par la cheute des Rois, et des Royaumes : et par la ruine des Republiques $\rangle^{17}$. Et le deuxième adjectif employé par le célestin pour caractériser les récits de ce volume, « pitoyable », même s'il est commun dans le récit bref dès le $\mathrm{XVI}^{\mathrm{e}}$ siècle, ne fait que préciser cet horizon d'attente classique, tout imprégné du pathos aristotélicien.

Dans le titre du troisième volume de ses récits Gonon utilise un seul adjectif : les histoires qu'il présente sont - ou doivent être - admirables. Même si dans les liminaires l'auteur garde à ce propos un silence significatif, ce critère est pourtant assez vaste. La nature des histoires admirables les oppose aux histoires curieuses à cause de la charge émotionnelle qu'elles doivent imposer aux lecteurs : lorsque les histoires curieuses entraînent, comme nous l'avons dit, une réaction modérée, les histoires admirables, elles, éveillent des sentiments bien plus forts. Car, étymologiquement, le mot « admirable » renvoie à la notion de " merveille » qui engendre l'étonnement dans l'esprit et qui, avec d'autres notions synonymiques, était

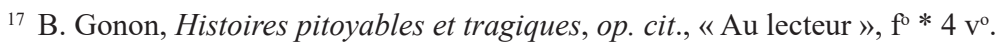


volontiers utilisée par les écrivains du temps ${ }^{18}$. Toutes ces épithètes, d'après Albert Pauphilet « trop générales et peu topiques $»^{19}$, expriment l'idée de l'admiration devant des choses fascinantes, susceptibles d'entraîner l'esprit, voire de l'envoûter. Les histoires admirables, écho lointain des recueils de Simon Goulart, promettent donc la rencontre de quelque chose d'inouï et de passionnant.

\subsection{Indications thématiques}

Les indications thématiques insérées dans les titres annoncent de façon générale le contenu des volumes; elles concernent la matière narrative et les dramatis personae. C'est ainsi que, dans le premier livre, Gonon évoque le genre d'événements que racontent ses histoires : y « sont representées les étranges avantures des personnes Illustres ». Soulignons quelques aspects connotés par ces mots : d'abord, l'idée de la représentation, spectacle sous-entendu ; ensuite une pluralité de faits singuliers, « aventures », précisément, donc ce qui advient au hasard ; qui plus est, ces « aventures » sont « étranges », elles échappent à notre horizon d'attente habituel, quotidien ; enfin, les protagonistes de ces aventures ce sont des « personnes Illustres », expression qui fait penser à des individus probablement dotés d'un certain pouvoir et ayant sans doute exercé une influence sur le cours de l'histoire.

Les titres des deux volumes suivants conservent l'idée de la représentation tout en en élargissant l'extension : on y découvre des « actions vertueuses et vicieuses de quelques illustres Payens et Chrestiens [qui] sont amplement representées ». On constate aussi qu'à la notion d' " aventures étranges », suscitant un certain intérêt à l'imagination et à l'intelligence, le célestin substitue celle d'« actions vertueuses et vicieuses », expression qui plonge les faits dans une dimension axiologique. Enfin, il précise ici ses protagonistes qui ne sont plus simplement connus pour leurs exploits, mais possèdent aussi un trait distinctif confessionnel : « païens et chrétiens $»^{20}$.

Le dernier volume se caractérise par un titre plus complexe qui réunit les éléments constitutifs des deux tomes précédents et en ajoute d'autres : «étranges avantures, vertus, vices et misères de plusieurs Empereurs, Roys, Princes, Grands Seigneurs, et autres Personnes considerables sont amplement representées ». C'est

${ }^{18}$ A. Lorian, Tendances stylistiques dans la prose narrative en France du XVI siècle, Paris, Klincksieck, 1973, p. 47.

19 A. Pauphilet, Études sur la Queste del Saint Graal, Paris, Honoré Champion, 1921, p. 187.

${ }^{20}$ Notons que Valère Maxime se sert de la même méthode dans ses Faits et dits mémorables où il présente les vices et les vertus aussi bien des Romains que des « barbares » : entre autres, les Gaulois ou les Égyptiens. Benoît Gonon connaissait le texte de Valère et le lien entre ces deux auteurs est facile à prouver. Certaines histoires, selon son aveu, sont empruntées à cet écrivain latin : « Histoire de Porcia, femme de Marcus Brutus » (Histoires pitoyables et tragiques, op. cit., p. 28-33) ; « Histoire d'Eustache de Sainct Pierre et de ses compagnons » (Histoires admirables, op. cit., p. 1-49); ou un épisode intercalé dans l'« Histoire de Louys de Bourbon » (ibid., p. 137). 
ainsi que reviennent ici les « étranges aventures » que complète un mot du registre tragique, «misères »; mais Gonon insère dans ce titre deux autres termes, désignant la disposition morale de l'individu au bien ou au mal, « vertus » et « vices ». À l'homogénéité des deux premiers titres succède ici une forme hétérogène qui investit non seulement l'imagination et l'intelligence du lecteur, mais aussi son sens moral et son affectivité. La composante thématique du titre de ce troisième volume constitue donc une captatio beneuolentiae singulière, réunissant le delectare et le mouere.

Somme toute, on peut noter que, d'un volume de récits à l'autre, Gonon amplifie les indications thématiques. Espère-t-il que la complexité du titre soit susceptible d'attirer un plus grand nombre de lecteurs ? Peut-être. En tout cas, les titres analysés contiennent un élément capable de leur plaire, à savoir la uarietas. En effet, l'ensemble de ces actions, aventures, etc. suppose une multiplicité de faits. Confrontés à des situations narratives diversifiées, les lecteurs pourront admirer avec admiration et plaisir la richesse du monde, créé par Dieu et recréé par l'écrivain.

\subsection{Destinateur}

La question du destinateur du titre semble au premier abord ne poser aucun problème. C'est bien évidemment Benoît Gonon qui a intitulé ses recueils, et son nom figure sur leurs pages de titre. Mais, à voir les choses de près, elles se compliquent un peu. En effet, l'auteur prétend que ses histoires ont été « recolligées / recueillies de plusieurs celebres Historiens / Autheurs ». Il est producteur du titre, mais non pas de la matière du livre. Il vend comme sien un objet qui ne lui appartient pas. Dans la perspective théorique, le statut du compilateur est par conséquent ambigu, au moins d'un point de vue moderne. Car si personne n'a l'idée de contester la paternité des titres du célestin, nombreux seraient ceux qui reprocheraient à ses recueils le larcin littéraire, le manque d'invention. Or, à la Renaissance dont il est héritier, la compilation est loin de mériter la disgrâce des auteurs et des lecteurs ${ }^{21}$. Dans une célèbre Lettre à Lucilius (84), Sénèque avait déjà pris pour modèle de l'écriture une abeille butinant les fleurs pour faire du miel ; le fruit de son labeur est juste un bouquet de fleurs qu'il ne fait que cueillir, écrit Simon Goulart à propos de son recueil ${ }^{22}$. Quant à Gonon, dans le paratexte des Histoires admirables, il développe sa propre métaphore du compilateur :

${ }^{21}$ Outre les compilations déjà citées de Simon Goulart et Pierre Boitel, citons à titre d'exemple celle de Pierre Messie (Les Diverses Leçons, Lyon, Barthelemy Honorat, 1577 ; éd. princeps Paris, 1552) et celle d'Antoine Du Verdier qui prétend être une suite de la précédente (Les Diverses Leçons d'Antoine du Verdier [...] suivans celles de Pierre Messie, Lyon, Barthelemy Honorat, 1577).

22 S. Goulart, Histoires admirables et memorables de nostre temps, t. II, Paris, Jean Houzé, 1610, « Epistre $», \mathrm{f}^{\mathrm{0}}$ ã ii $\mathrm{v}^{\mathrm{o}}$. 
Et comme il n'est permis de blasmer l'Orfevre, qui ne met en œuvre, que l'or, \& la pierre precieuse, qu'on luy a donné, sans y adjouster que son travail, \& ses outils : \& qu'il n'est bien seant de casser le Miroir, qui represente ce qu'on luy oppose ; aussi je ne dois estre blasmé, si dans ces Histoires se trouve quelque manquement, veu qu'en les escrivant, je n'y ay employé que mon travail, \& n'ay representé que les choses que j'ay trouvées dans les bons $\&$ approuvez Autheurs, desquels je me suis servy pour l'embellissement de mon sujet ${ }^{23}$.

Il faudrait donc renverser l'idée de Gérard Genette ${ }^{24}$ et affirmer qu'explicitement Gonon est un « destinateur de fait » de ses titres, mais que, implicitement, il n'en est pas le « destinateur de droit». Par ailleurs, en empruntant ses histoires à une pluralité d'écrivains reconnus, notre religieux renforce son souci de la uarietas qu'il a déjà exprimé dans les indications thématiques de ses titres. L'emprunt est enfin un moyen commode pour s'approprier la parole des autres et, comptant sur leur véridicité, espérer trouver un accueil bienveillant auprès des censeurs qui légitiment la publication de l'œuvre ${ }^{25}$.

\subsection{Destinataire}

Nous avons observé plus haut que, par le terme d'histoires, Gonon s'inscrit dans la tradition du récit bref. Il faut maintenant ajouter que ce terme lui permet aussi de rattacher ses recueils à l'historiographie. Le célestin avoue explicitement son inspiration. Dans l'avis «Au lecteur » des Histoires admirables, il place une référence directe au topos cicéronien de l'histoire, magistra uitae d'un chacun ${ }^{26}$.

Ciceron Prince de l'Eloquence Latine, au second livre de l'Orateur, traictant de l'Histoire, l'appelle tesmoing des temps, lumiere de verité, vie de la memoire, regente de la vie humaine, et messagere de l'antiquité ; De mesme l'Histoire (que Ciceron appelle Miroir de la vie humaine) non seulement divertit, et resjouyt : ains aussi represente les vices et vertus.

${ }^{23}$ B. Gonon, Histoires admirables, op. cit., «Au lecteur », fo $4 \mathrm{v}^{\circ}$. Une métaphore similaire est présentée par l'auteur dans le préliminaire à sa Chasteté recompensée (Bourg en Bresse, Jean Tainturier, 1643, fo $4 \mathrm{v}^{\circ}$ ) : « Encor qu'une pierre precieuse porte son prix \& valeur avec soy, que la prodigue mere Nature luy a donné en la produisant \& formant : toutesfois elle se rend plus riche $\&$ estimable, quant elle est mise en œuvre par l'Orfevre judicieux. De mesme à mon jugement, encore que les actions vertueusement faites, soient d'elles-mesmes dignes de grande louange : si est-ce qu'elles acquierent un lustre, \& ornement beaucoup plus exquis, quant le gracieux labeur des Escrivains les daigne recueillir pour les consacrer à l'immortalité : et les enchassant au temple de l'heureuse memoire, les fait servir de notables reliques à la posterité ».

${ }^{24}$ G. Genette, op. cit., p. 71.

25 À double reprise, Gonon reçoit effectivement un avis favorable à la publication de ses recueils. Voir les «Approbations des docteurs » dans les Histoires veritables et curieuses et dans les Histoires admirables.

${ }^{26}$ B. Gonon, Histoires admirables, op. cit., « Au lecteur », fo 4 r ${ }^{\circ}$. 
Cette inscription du récit bref dans l'historiographie, théorisée déjà par Bénigne Poissenot dans le Prologue à ses Nouvelles histoires tragiques, annonce tacitement la visée didactique, moralisatrice des récits gononiens. L'auteur écrit «non seulement pour recreer les Lecteurs : ains aussi pour les provoquer à detester le vice, et embrasser la vertu »; et il poursuit :

Mon intention n'a pas esté de publier ces Histoires pour repaistre seulement l'esprit d'une folle curiosité ; ou pour y apprendre seulement quelque chose, dont puis après on en puisse babiller par vaine ostentation : mais je desire qu'on s'y porte avec telle consideration, qu'il n'y a en icelles fait ou evenement de quelque chose que ce soit, que chacun ne la puisse, et doive prendre pour en faire son profit ${ }^{27}$.

Or, dans le titre de son troisième volume, il ajoute une information supplémentaire, bien claire : ses récits ont été rédigés " pour l'instruction et divertissement de toutes sortes de personnes ». Se trouve ainsi formulé le docere que Gonon estime tout particulièrement et qui est au centre des préoccupations humanistes. Mais le célestin y signale aussi, par une expression très générale, le public qu'il vise. Cette tournure n'était-elle cependant pas trop vague pour intriguer ceux qui auraient manqué de happer les deux volumes de récits précédents ? Nous l'ignorons. Quoi qu'il en fût, force est de signaler que Gonon témoigne un souci manifeste de son destinataire. Plusieurs arguments semblent le prouver. Le recours aux autorités, pour conventionnel qu'il soit dans le discours humaniste, n'en permet pas moins d'accréditer sa propre parole et de présenter au public un écrivain digne de foi : "Aussi ces Histoires, ayans esté extraites des bons Autheurs, ne pourront estre que bien receues de tous les Lecteurs $»^{28}$. La structure de plus en plus complexe de ses titres révèle que, le temps passant, notre religieux se fait de plus en plus attentif à ce paratexte : en l'amplifiant, il entend en augmenter la force persuasive. Enfin, rappelons-le, dès 1637 le célestin n'écrit plus qu'en français, en ayant abandonné le latin pour s'ouvrir à un lectorat plus vaste.

Les récits brefs de Benoît Gonon avaient-ils un pouvoir de séduction sur les lecteurs de son époque ? Rien ne permet de l'affirmer. Nous savons en revanche que, vers le milieu du XVII ${ }^{e}$ siècle, la mode du récit humaniste, incarné entre autres par les histoires tragiques à la Boaistuau et Belleforest ou par les histoires admirables à la Goulart, est en train de s'éteindre; que la mort emporte Jean-Pierre

${ }^{27}$ B. Gonon, Histoires veritables et curieuses, op. cit., "Au lecteur », fo $5 \mathrm{r}^{\circ}$. Voir aussi : " Cette consideration m'a donné sujet d'escrire ces Histoires, capables non seulement de divertir les Lecteurs, ains aussi de les porter à la vertu » (idem, Histoires pitoyables et tragiques, op. cit., «Au lecteur », $\left.4 \mathrm{r}^{\circ}\right)$.

${ }^{28}$ B. Gonon, Histoires admirables, op. cit., « Au lecteur », $\mathrm{f}^{\circ} 5$ r ${ }^{\circ}$. 
Camus, le plus fécond fournisseur de recueils d'histoires dissimulées derrière des titres alléchants ; et que, avec Les Nouvelles françaises de Charles Sorel (1623), consacrées exclusivement à l'amour et sujettes à d'importantes amplifications, vient petit à petit le goût pour la nouvelle longue, voire romanesque, souvent publiée isolément et non plus en recueils ${ }^{29}$. Dans ce climat littéraire, Gonon cherchait sans doute sa propre voie sans dissimuler qu'au point de vue littéraire il était un enfant de la Renaissance. Il croyait pouvoir trouver son chemin à l'aide d'une poignée d'éléments topiques manifestes dans les recueils de nouvelles humanistes antérieurs, conscient qu'il devait être de l'originalité qu'il apportait en combinant ces topö̈ entre eux. Et nous pouvons supposer qu'avec la uarietas, croissant d'un volume à l'autre, qu'il a assurée à ses titres de recueils il espérait intéresser quelque public. Enfin, avançons pour terminer qu'il serait également opportun de s'interroger sur la fidélité avec laquelle Gonon réalise son programme esquissé dans les titres des livres en question - mais c'est ici matière pour un autre article.

\section{Bibliographie}

Becquet, Antoine, Gallicae Celestinorum congregationis ordinis $S$. Benedicti monasterium fundationes uirorumque uita aut scriptis illustrum, elogia historica, Paris, Florentinum Delaulne, 1749

Cremona, Nicolas "Pleines de chair et de sang " : poétique d'un " genre à succès ", l'histoire tragique, thèse de doctorat, Paris, 2009

Depéry, Jean-Irenée, Biographie des hommes célèbres du département de l'Ain, Bourg, P.-F. Boittier, 1833

Duchet, Claude, "La Fille abandonnée et La Bête humaine, éléments de titrologie romanesque », Littérature, Année 1973, nº 12, p. 49-73 https://doi.org/10.3406/litt.1973.1989

Genette, ${ }^{\mathrm{G}}$ érard, Seuils, Paris, Éditions du Seuil, 1987

Godenne, René, Histoire de la nouvelle française aux XVII et XVIII siècles, Genève, Droz, 1970

Gonon, Benoît, Histoires veritables et curieuses, où sont representées les étranges avantures des personnes Illustres : Recolligées de plusieurs celebres Historiens : Par le R. Pere Benoist Gonon, Celestin de Lyon, Lyon, Jaques du Creux, 1644

Gonon, Benoît, Histoires pitoyables et tragiques, où les actions vertueuses et vicieuses de quelques illustres Payens et Chrestiens sont amplement representées. Recueillies de plusieurs celebres Historiens. Par le R. P. Benoist Gonon, Celestin de Lyon, Lyon, Claude de la Rivière, 1646

Gonon, Benoît, Histoires admirables, où les étranges avantures, vertus, vices et misères de plusieurs Empereurs, Roys, Princes, Grands Seigneurs, et autres Personnes considerables sont amplement representées. Pour l'instruction et divertissement de toutes sortes de personnes. Recueillies de plusieurs celebres Autheurs, par le Reverend Pere Benoist Gonon, Celestin de Lyon, Lyon, Claude de la Rivière, 1653

Goulart, Simon, Histoires admirables et memorables de nostre temps, t. II, Paris, Jean Houzé, 1610, «Epistre », $\mathrm{f}^{0}$ ã ii $\mathrm{v}^{0}$

Hainsworth, Georges, Les "Novelas exemplares » de Cervantes en France au XVII siècle », Paris, Champion, 1933

${ }^{29}$ Voir la bibliographie dans R. Godenne, op. cit., p. 250 sqq, et 300 sqq. Sur l'évolution de la nouvelle au XVII' siècle, voir ibid., p. 25-129. 
Hoek, Leo H., La marque du titre : dispositifs sémiotiques d'une pratique textuelle, La Haye, Mouton éditeur, coll. «Approaches to semiotics », 1981 https://doi.org/10.1515/9783110822786

Kasprzyk, Krystyna, Nicolas de Troyes et le genre narratif en France au XVI e siècle, Warszawa / Paris, Państwowe Wydawnictwo Naukowe / Librairie C. Klincksieck, 1963

Lorian, Alexandre, Tendances stylistiques dans la prose narrative en France du XVI siècle, Paris, Klincksieck, 1973

Pauphilet, Albert, Études sur la Queste del Saint Graal, Paris, Honoré Champion, 1921, p. 187

Pietrzak, Witold Konstanty, Le Tragique dans les nouvelles exemplaires en France au XVI siècle, Łódź, Wydawnictwo Uniwersytetu Łódzkiego, 2006

Poli, Sergio, Storia di storie. Considerazioni sull'evoluzione della storia tragica in Francia dalla fine delle guerre civili alla morte di Luigi XIII, Genova, Publicazioni dell'Istituto di Lingue e letterature straniere moderne, 1985

Thomas d'Aquin, Somme théologique, IIa IIae, trad. E. Belin, Paris, Librairie Ecclésiastique et Classique, 1856, question 167

Żywoty y nauki świętych pustelników zachodnich przez wielebnego oyca Benedykta Gonona Burgensa oyców Celestinów zakonnika wydane y zebrane a językiem tacińskiem napisane; manuscrit 2958/1, Dolnośląska Biblioteka Cyfrowa.

Marta Elżbieta Trębska a fait des études de philologie romane et de philologie classique à l'Université de Łódź. Depuis 2018, elle prépare une thèse de doctorat dans le programme de cotutelle sous la direction du prof. Witold Konstanty Pietrzak (Université de Łódź) et de la prof. Nathalie Grande (Université de Nantes). Sa thèse concerne Les Occurrences Remarquables de Jean-Pierre Camus parues en 1628 et consiste dans l'édition critique de ce texte. Ses intérêts scientifiques se concentrent autour des nouvelles exemplaires des XVI ${ }^{\mathrm{e}}$ et XVII ${ }^{\mathrm{e}}$ siècles, de la réception de l'Antiquité dans la littérature de l'Ancien Régime et des histoires dévotes de Jean-Pierre Camus.

\begin{tabular}{|c|c|}
\hline (cc)creative & $\begin{array}{l}\text { (C) by the author, licensee Łódź University - Łódź University Press, } \\
\text { Łódź, Poland. This article is an open access article distributed under } \\
\text { the terms and conditions of the Creative Commons Attribution license } \\
\text { CC-BY-NC-ND } 4.0 \text { (https://creativecommons.org/licenses/by-nc-nd/4.0/) }\end{array}$ \\
\hline & Received: 2019-04-19; Accepted: 2020-12-20 \\
\hline
\end{tabular}

